\title{
Thymoma by AJCC v8 Stage
}

National Cancer Institute

\section{Source}

National Cancer Institute. Thymoma by A/CC v8 Stage. NCI Thesaurus. Code C136345.

A term that refers to the staging of thymoma, following the rules of the TNM AJCC v8 classification system. 